\title{
Wieviel Psychologie steckt in der Psychotherapie?
}

\author{
Hans-Ulrich Wittchen ${ }^{a}$ b Winfried Rief $^{c}$ \\ ${ }^{a}$ Institut für Klinische Psychologie und Psychotherapie, Technische Universität Dresden, Dresden, Deutschland; \\ ${ }^{b}$ Center of Epidemiology and Longitudinal Studies (CELOS), Technische Universität Dresden, Dresden, Deutschland; \\ ${ }^{\mathrm{c}}$ AG Klinische Psychologie und Psychotherapie, Philipps-Universität Marburg, Marburg, Deutschland
}

Unter diesem Motto steht der 9. Workshopkongress 2015 der Fachgruppe Klinische Psychologie und Psychotherapie der Deutschen Gesellschaft für Psychologie. Wir freuen uns, dass es uns gelungen ist, zu diesem Anlass alle Keynote-Vortragenden für die Idee zu gewinnen, Ihre Kernaussagen als Beitrag in der VerHALTENSTHERAPIE zu publizieren. Zugleich danken wir dem KargerVerlag für die Bereitschaft, unsere Idee unter großem Zeitdruck zu realisieren.

Zweifellos leben wir in aufregenden Zeiten! Nicht nur, was die Entwicklung der Psychologie und speziell der Verhaltenstherapie als genuin psychologisches Verfahren angeht, sondern auch, was die Psychotherapie als Ganzes betrifft.

Wir erleben eine sich exponentiell beschleunigende wissenschaftliche Entwicklung hinsichtlich der Ursachenforschung zu psychischen Störungen wie auch der interventionellen und translationalen Forschung. Diese hat in vielen Bereichen das Potenzial, zukünftig die Versorgung von Menschen mit psychischen Problemen hinsichtlich Prävention, Therapie und Rehabilitation zu verbessern. Hierzu geben die Beiträge von Wittchen et al., [2015], Flor [2015], Weisz [2015] und Craske [2015] in dieser Ausgabe der Verhaltenstherapie vielfältige und konkrete Beispiele.

Zugleich erleben wir aber auch, wie schwer wir uns tun, die offensichtlich immer größere Kluft zwischen Wissenschaft und Praxis zu schließen. Trotz aller Bemühungen, die in zahlreichen Leitlinien und Metaanalysen bestätigten State-of-the-ArtMethoden der Kognitiven Verhaltenstherapien (KVT) flächendeckend und qualitätsgesichert in der Routineversorgung verfügbar zu machen, scheint sich an der insgesamt unbefriedigenden Situation der Psychotherapiepraxis offensichtlich nur wenig $\mathrm{zu}$ ändern. Psychotherapien in der Versorgung sind oftmals länger als notwendig (was angesichts langer Wartezeiten auf Behandlungsplätze ethisch problematisch ist) und orientieren sich nicht ausreichend an empirischen Gesetzmäßigkeiten, wie Veränderungen in der Psychotherapie effektiv und ökonomisch erreicht werden können. Zwar teilen wir dieses offensichtlich notorische Problem, neue wissenschaftliche Erkenntnisse schnell und qualitätsgesichert in die Versorgung zu bringen, mit dem gesamten Medizinbereich. Allerdings weist der Psychotherapiesektor doch eine Reihe von Besonderheiten auf, die bei der Suche nach Lösungen zu beachten sind. Hierzu liefert der Beitrag von Bohus [2015] eine Reihe von höchst anregenden Denkimpulsen, die deutlich machen, wie auch diverse persönliche Motive Veränderungsprozesse blockieren. Darüber hinaus ist es uns, als Herausgeber bzw. Gastherausgeber dieser Ausgabe von Verhaltenstherapie ein wichtiges Anliegen, darauf hinzuweisen, dass die Kluft zwischen Wissenschaft und Praxis in der Psychotherapie auch zum Teil durch Identitätsprobleme sowie durch eine hohe Bereitschaft der Orientierung an traditionellem Denken bei fehlender Bereitschaft, Neues aufzugreifen, mitbedingt wird.

Gerade im Zuge der Diskussion um eine Novellierung der Psychotherapeutenausbildung stellt sich die Frage, was eigentlich unsere Mutterwissenschaft ist. Sind beziehungsweise empfinden sich Psychologische Psychotherapeuten noch als Psychologen? Fühlen sie sich noch ihrer "Mutterwissenschaft», der Psychologie, verpflichtet und verbunden? Oder haben sie als Psychologische Psychotherapeuten aufgrund der rechtlichen Rahmenbedingungen und der «Verkammerung» eine neue primäre Identität als «Psychotherapeuten» und die Bindung zur Psychologie verloren oder gar bewusst aufgegeben? Unsere ärztlichen Psychotherapeutenkolleginnen und -kollegen scheinen nicht gleichermaßen von dieser Identitätsfrage betroffen zu sein; sie bleiben ihrer medizinischen Fachdisziplin offensichtlich näher.

Wir als Fachvertreter für Klinische Psychologie und Psychotherapie sehen naturgemäß die Psychologie mit allen ihren Teilfächern als die essenzielle «Mutterwissenschaft» der Psychotherapie an. Eine so junge und sich dynamisch entwickelnde Fachdisziplin braucht eine transparente akademische Fachverortung, um angesichts der noch unbefriedigenden wissenschaftlichen Fundierung für die Zukunft gerüstet zu sein.

\section{KARGER}

Fax +497614520714

\section{(c) 2015 S. Karger GmbH, Freiburg}

$1016-6262 / 15 / 0252-0090 \$ 39.50 / 0$
Prof. Dr. Winfried Rief

AG Klinische Psychologie und Psychotherapie

Fachbereich Psychologie, Philipps-Universität Marburg

Gutenbergstraße 18, 35032 Marburg, Deutschland

rief@staff.uni-marburg.de 
Aber diese Einschätzung wird, wie vor allem die Diskussion zur Novellierung des Psychotherapeutengesetz zeigt, nicht unbedingt von allen Vertretern der Psychotherapeutenkammern und von allen Psychotherapeuten geteilt, die ja oft aus historischen und curricularen Gründen verständlicherweise eine andere Perspektive haben. Aber das Festhalten an alten Konzepten bestimmter Traditionen sowie an alten Aus- und Weiterbildungsstrukturen bagatellisiert oftmals bestehende Probleme. Darauf weist auch der zusätzlich mit aufgenommene Beitrag von Tripp et al. [2015] zur psychotherapeutischen und psychiatrischen Aus- und Weiterbildung hin, der deutlich macht, dass bei allem Stolz auf Aus- und Weiterbildungsqualität hier sowohl im ärztlichen als auch im psychologischen Bereich deutlicher Raum für Verbesserungen ist.

Wir hoffen, dass diese Ausgabe der Verhaltenstherapie nicht nur als weitere Bereicherung und Stimulanz für eine lebendige Diskussion im Rahmen des Workshopkongresses in Dresden aufgegriffen wird, sondern auch darüber hinaus zur Reflexion der eigenen Identität und Weiterentwicklung der fachlichen Perspektiven von Klinischer Psychologie und Psychotherapie beiträgt.

\section{Literatur}

Bohus M: Elfenbeintürme im Treibsand oder: Was macht es so schwierig, Erkenntnisse aus der Forschung in der therapeutischen Praxis umzusetzen? Verhaltenstherapie 2015;25:145-155.

Craske M: Optimizing exposure therapy for anxiety disorders: an inhibitory learning and inhibitory regulation approach. Verhaltenstherapie 2015;25:134-143.
Flor H: Lost in translation: Psychologische Mechanismen und Psychotherapie. Verhaltenstherapie 2015;25:111117.

Tripp J, Schweiger U, Ströhm W, Stromberg C, Quaschner K: Qualität in der Weiterbildung: Aktueller Stand und Zukunft psychotherapeutischer Aus- und Weiterbildung. Verhaltenstherapie 2015;25:119-126.
Weisz JR: Bridging the research-practice divide in youth psychotherapy. Verhaltenstherapie 2015;25:129-132.

Wittchen H-U, Härtling S, Hoyer J: Psychotherapy and mental health as a psychological science discipline. Verhaltenstherapie 2015;25:98-109. 